\title{
Defect related switching field reduction in small magnetic particle arrays
}

\author{
M. J. Donahue \\ National Institute of Standards and Technology, Gaithersburg, Maryland 20899 \\ G. Vértesy \\ Research Institute for Technical Physics and Materials Science, Hungarian Academy of Sciences, \\ H-1525 Budapest, P.O.B. 49, Hungary
}

M. Pardavi-Horvath

The George Washington University, Department of ECE, Washington, D.C. 20052

(Presented on 13 November 2002)

\begin{abstract}
An array of $42 \mu \mathrm{m}$ square, $3 \mu \mathrm{m}$ thick garnet particles has been studied. The strong crystalline uniaxial anisotropy of these particles results in the stable remanent state being single domain with magnetization parallel to the film normal. Magneto-optic measurements of individual particles provide distribution statistics for the easy-axis switching field $H_{\mathrm{sw}}$, and the in-plane hard-axis effective anisotropy field, $H_{\text {eff }}$, which induces the formation of a metastable stripe domain structure. Both $H_{\text {sw }}$ and $H_{\text {eff }}$ are much smaller than the crystalline anisotropy field. Micromagnetic simulations show that the small $H_{\text {sw }}$ cannot be attributed to shape anisotropy, but is consistent with smooth, localized reductions in the crystalline anisotropy caused by defects in either the particles or the substrate. () 2003 American Institute of Physics. [DOI: 10.1063/1.1557399]
\end{abstract}

\section{INTRODUCTION}

The broad statistical distribution of switching fields of small, nonellipsoidal shape particles in patterned magnetic media is an important concern for future high density magnetic recording, MRAM and sensor arrays, and magnetic MEMS.

Hysteresis loop measurements on individual particles of a model system of a 2D array of single domain, single crystal garnet particles have shown that the measured statistical distribution of switching fields, $H_{\text {sw }}$, has a large standard deviation, $\quad H_{\mathrm{sw}}=22.7 \mathrm{kA} / \mathrm{m} \pm 6.8 \mathrm{kA} / \mathrm{m} \quad(285 \mathrm{Oe} \pm 85 \mathrm{Oe})$, with the highest measured $H_{\mathrm{sw}}(\max ) \approx 50 \mathrm{kA} / \mathrm{m}(600 \mathrm{Oe}){ }^{1}$ Some of the overall reduction of the average switching field, relative to the anisotropy field of the whole $1 \mathrm{~cm}^{2}$ chip, $H_{u}$ $=170 \mathrm{kA} / \mathrm{m}(2100 \mathrm{Oe})$, was related to the nonuniform magnetization due to the shape and size of the particles. However, the shape effect cannot explain the broad distribution of the switching fields of individual particles. The original single crystalline magnetic garnet film has an exceptionally high crystalline quality, with a very low dislocation density, no inclusions, and no visible edge, corner, or surface defects on the individual particles. Further experimental investigations have shown that there are very weak crystalline defects, revealed only by magnetic defect decoration experiments, responsible for the local reduction and the broad distribution of the switching fields. ${ }^{2}$

It is expected that the reduction of $H_{\mathrm{sw}}$ is due to localized crystalline defects, reducing the effective anisotropy field. In the present work statistical data were collected on the effective anisotropy field distribution of several hundred individual particles along the hard axis, and compared to the easy axis switching field distribution.

Numerical 3D micromagnetic modeling confirms that the overall reduction of the switching field, relative to the crystalline anisotropy field, cannot be explained by the particle shape demagnetizing field. The switching field and effective anisotropy field reduction is consistent with a model that includes regions of reduced anisotropy caused by defects, in either the particles or the substrate, with the defect's stress induced anisotropy field extending into the particle. ${ }^{3-5}$

These results have a direct consequence for the case of small metallic particles, where there is a much higher probability for random defects, such as grain boundaries, inclusions, substrate-film interface effects, and dislocation densities and associated stress fields that are much higher than in this model system.

\section{EXPERIMENTS}

The experiments were performed on a regular 2D square array of particles, etched into a single crystalline magnetic garnet film (substituted $\mathrm{Y}_{3} \mathrm{Fe}_{5} \mathrm{O}_{12}$ ), grown epitaxially on a nonmagnetic GGG $\left(\mathrm{Gd}_{3} \mathrm{Ga}_{5} \mathrm{O}_{12}\right)$ substrate. The size of the particles is $42 \mu \mathrm{m} \times 42 \mu \mathrm{m} \times 3 \mu \mathrm{m}$, separated by $12 \mu \mathrm{m}$ wide grooves. The $5 \mathrm{~mm}$ square sample contains about $10^{4}$ particles. Garnet crystals are extremely brittle, and the energy of dislocation formation is very high, such that large garnet boules are regularly grown with a dislocation density of less than $10 \mathrm{~cm}^{-3}$. SEM observations show the high quality of the edges and corners of the etched particles.

The mean value of the uniaxial anisotropy field, $H_{u}$, was determined on the patterned film from differences between the middle slopes of saturation hysteresis loops, measured with the external field oriented in the parallel and perpendicular directions to the easy axis. The same result was obtained using both the vibrating sample magnetometer (VSM) and magneto-optical methods. The magnetization of the sample, measured using VSM and SQUID, was found to be $12.7 \mathrm{kA} / \mathrm{m}\left(4 \pi M_{s}=160 \mathrm{G}\right)$, which is very low compared 


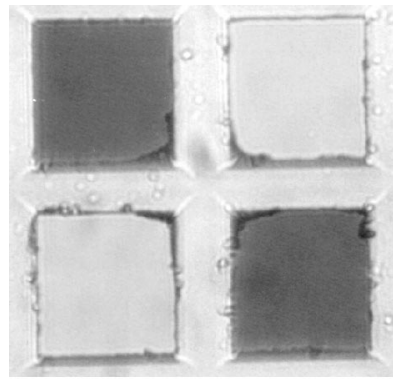

(a)

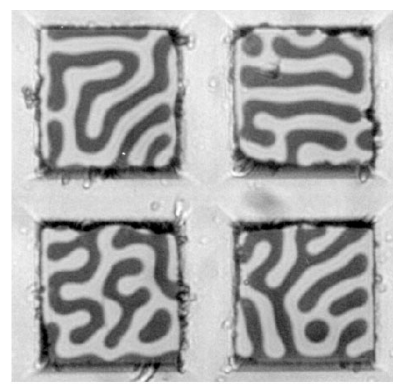

(b)
FIG. 1. (a) Demagnetized state of 4 garnet particles on a 2D array, having two stable states of magnetization, "dark" and "light" (checkered pattern); (b) Metastable demagnetized state of the same four particles (stripe domain structure). Particle size: $42 \mu \mathrm{m} \times 42 \mu \mathrm{m} \times 3 \mu \mathrm{m}$.

to the uniaxial anisotropy field $H_{u}$. As a result, the particles have strong uniaxiality, characterized by $Q=H_{u} / 4 \pi M_{s}$ $>10$, ensuring that the particles are single domain, having only two stable magnetic states, either "up" or "down" along the easy axis, normal to the film plane. Each particle has a rectangular hysteresis loop, and the switching of the whole system proceeds by consecutive switching of individual particles. The particles were found to be only weakly interacting. ${ }^{6}$

Hysteresis loops of individual particles were measured magnetooptically (MO) in the Faraday geometry, with simultaneous visual observation of switching events. The composition of the garnet is optimized to have a very high Faraday effect, leading to a very high contrast in MO measurements. Switching fields along the easy axis have been measured earlier. $^{7}$ Each measurement was started from the saturated state along the easy axis.

The effective anisotropy field of many individual particles was systematically measured magneto-optically, by focusing the light beam on a single particle. Before each measurement the particles were saturated in a normal magnetic field, parallel to the easy axis. Then an in-plane magnetic field was increased from zero, and the field at which stripes were nucleated was detected as a sudden change in the light intensity vs magnetic field curve.

The stripe domain state is the ground state of the magnetically soft epitaxial garnet layer before it is structured into the 2D array. Magnetization proceeds in this case by domain wall motion. In the structured state, however, the stable demagnetized state is the "checkered" pattern shown in Fig. 1(a), where the magnetization of each individual particle is oriented along the easy axis. The stripe domain pattern, displayed in Fig. 1(b), is obtained by application of an in-plane field. This state is only metastable; application of a moderate field of $6 \mathrm{kA} / \mathrm{m} \pm 0.2 \mathrm{kA} / \mathrm{m}$ ( $75 \mathrm{Oe} \pm 3 \mathrm{Oe}$ ) normal to the film plane collapses the stripes and saturates the material. Reversing the normal field from this point switches the individual particles one by one, eventually reaching again the stable demagnetized state of Fig. 1(a).

\section{RESULTS}

The distribution of the switching fields for 370 particles, measured as the half-width of the easy-axis hysteresis loop

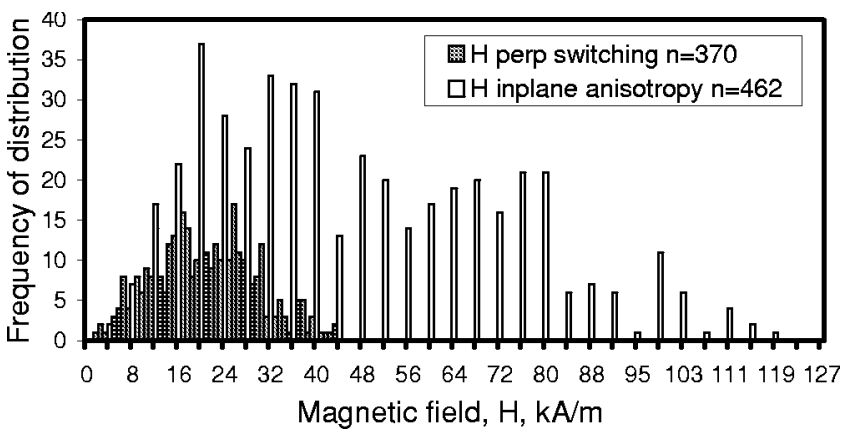

FIG. 2. Distribution of the switching field along the easy axis, normal to the particle ( $n=370$ particles), and of the effective anisotropy field in-plane, along the hard axis $(n=462)$.

of individual particles, and the distribution of the effective anisotropy field, measured as the hard axis saturating field on 462 individual particles, are shown in Fig. 2. The switching field distribution for this set of particles has a mean value $H_{c}=20 \mathrm{kA} / \mathrm{m}(250 \mathrm{Oe})$, with a standard deviation of 10.4 $\mathrm{kA} / \mathrm{m}$ (131 Oe). The highest measured switching field is 44 $\mathrm{kA} / \mathrm{m}(550 \mathrm{Oe})$, in agreement with our previous data on similar samples.

It is expected that the reduction of $H_{\mathrm{sw}}$ is due to localized crystalline defects, causing a reduced effective anisotropy field. Our previous measurements revealed very good quantitative correlation between the strength of the defectdomain wall interaction, and the magnitude of the switching field. ${ }^{2}$ Defects reduce the local nucleation field, thus decreasing the switching field of the particles. Figure 2 shows that the distribution of $H_{\text {eff }}$ is much broader than the switching field distribution, and the shape of the distribution differs from that of $H_{\mathrm{sw}}$. There is a broad high field component of $H_{\text {eff }}$ with the highest observed value above $120 \mathrm{kA} / \mathrm{m}(1500$ Oe).

The highest switching field, $H_{\mathrm{sw}}(\max )$, is much lower than $H_{\text {eff }}(\max )<H_{u}$. This observation shows that the reduction of the switching field is most dramatic for very strong defects having low $H_{\text {eff }} \leqslant H_{c}$. However, reduction of the switching field due to weaker defects is also observed. This implies that any defect can be detrimental for a small magnetic device, reducing the characteristic field by a disproportionately large amount.

\section{MICROMAGNETIC MODELING}

Computer simulations were performed to help interpret the experimental results. We used the public domain OOMMF micromagnetic package ${ }^{8}$ for these simulations. The values used for material parameters $\left(M_{s}=12.7 \mathrm{kA} / \mathrm{m}, K_{u}\right.$ $=1333 \mathrm{~J} / \mathrm{m}^{3}, A=3 \times 10^{-12} \mathrm{~J} / \mathrm{m}$ ) were representative of the experimental samples. The simulation discretization cells were $15.625 \mathrm{~nm}$ cubes, which is one-third the magnetocrystalline-exchange length $\sqrt{A / K}=47 \mathrm{~nm}$, and less than one-tenth the magnetostatic-exchange length $\sqrt{A /\left(0.5 \mu_{0} M_{s}^{2}\right)}=170 \mathrm{~nm}$. The stopping criterion for identification of equilibrium states was $|\mathbf{M} \times \mathbf{H}|_{\text {sup }} / M_{s}^{2}<0.008$.

To gauge the importance of shape anisotropy in the switching mechanism, simulations were performed on 
defect-free, $\quad 2 \mu \mathrm{m} \times 2 \mu \mathrm{m} \times 500 \mathrm{~nm}$ and $4 \mu \mathrm{m} \times 4 \mu \mathrm{m}$ $\times 125 \mathrm{~nm}$ models. To break the problem symmetry, the applied field was aligned $1^{\circ}$ off the film normal. The switching fields were found to be approximately $145 \mathrm{kA} / \mathrm{m}$ (1820 Oe) and $140 \mathrm{kA} / \mathrm{m}$ (1760 Oe), respectively. These results are in close agreement with results predicted by the StonerWohlfarth uniform magnetization model for particles with these geometries. However, the micromagnetic simulations show that the switching process initiates by nucleation of a reversed, cylindrical domain in the middle of the particle, and proceeds by radial domain growth from the center towards the edges of the sample. Little variation in the magnetization along the film normal axis was detected, presumably because the demagnetization field is relatively uniform in the central region of these films. Additional thin-film simulations were performed on smaller models, and in all cases the switching field agreed with the Stoner-Wohlfarth estimate. Although the reversal as a whole is not by uniform rotation, the central regions essentially behave as StonerWohlfarth islands.

Simulations were also performed where the problem symmetry was broken by canting the crystalline easy axis away from the film normal. Reversal was found to proceed in the same manner as described above, with switching fields agreeing with Stoner-Wohlfarth calculations.

Although the experimental particles are too large to simulate directly, the apparent scale independence of these results suggests that they would hold for that system as well. The switching fields predicted by the above model are close to an order of magnitude larger than the average switching field observed experimentally. Since the defect-free simulations cannot explain the experimental results, we performed simulations on particles with isolated defects. The defects were modeled as localized reductions in the anisotropy constant $K_{u}$. In the first, simple defect model, we introduced a small region with reduced $K_{u}=K_{u \text {, defect }}$ into the interior of the sample, with a sharp transition to $K_{u \text {,bulk }}$ at the boundary of the defect. If this region was larger than about $500 \mathrm{~nm}$, then the magnetization in the defect was found to reverse at the anisotropy field in the defect. However, the region of reversed magnetization did not expand beyond the confines of the defect region if the applied field was smaller than $\left(H_{u \text {, bulk }}-H_{u \text {, defect }}\right) / 4$. In this case, the reversal was found to be a two step process: (1) nucleation of a reversed domain inside the defect, and (2) propagation of the reversed domain throughout the sample. This problem has been studied analytically in the case of a $1 \mathrm{D}$ domain wall. ${ }^{9}$ While the geometric structure of the defect is expected to have some effect, our simulations were in good agreement with the results of the $1 \mathrm{D}$ study.

In the simple defect model, the lowest attainable magnetization reversal field for the particle as a whole occurs when the nucleation field and the propagation field are equal, i.e., at one-fifth the bulk anisotropy field. Since $H_{u \text {,bulk }}$ $=170 \mathrm{kA} / \mathrm{m}$ in the experimental sample, the smallest switching field allowed by this model is $34 \mathrm{kA} / \mathrm{m}$, significantly larger than the average experimentally observed switching field $(20 \mathrm{kA} / \mathrm{m})$. However, the propagation field can be reduced by replacing the anisotropy step with a continuous transition region, as would be expected for a stress induced anisotropy field decaying with distance from, for example, an inclusion in the substrate. For simplicity, we modeled this transition as a linear ramp. ${ }^{10}$ The propagation field then depends upon the slope of the ramp. We found a slope of 1.2 $\times 10^{9} \mathrm{~J} / \mathrm{m}^{4}$ sufficiently gradual to produce a propagation field below $20 \mathrm{kA} / \mathrm{m}$, corresponding to a transition length of $1 \mu \mathrm{m}$ in the experimental samples. It appears that this length may be shortened by increasing the slope as one moves up the ramp, but examination of this is left for future research.

\section{ACKNOWLEDGMENTS}

One of the authors (G.V.) is supported by the Hungarian Scientific Research Fund through Projects Nos. T-026153 and T-035264.

${ }^{1}$ M. Pardavi-Horvath, G. Vértesy, B. Keszei, Z. Vértesy, and R. D. McMichael, IEEE Trans. Magn. 35, 3871 (1999).

${ }^{2}$ G. Vértesy and M. Pardavi-Horvath, Physica B 306, 251 (2001)

${ }^{3}$ M. Pardavi-Horvath, in Progress in Crystal Growth and Characterization (Pergamon, London, 1982), Vol. 5, pp. 175-220.

${ }^{4}$ M. Pardavi-Horvath, IEEE Trans. Magn. 21, 1694 (1985).

${ }^{5}$ G. Vértesy, M. Pardavi-Horvath, I. Tomas, and L. Pust, J. Appl. Phys. 63, 1694 (1988)

${ }^{6}$ M. Pardavi-Horvath and G. Vértesy, IEEE Trans. Magn. 30, 124 (1994).

${ }^{7}$ M. Pardavi-Horvath, IEEE Trans. Magn. 32, 4458 (1996).

${ }^{8}$ M. J. Donahue and D. G. Porter, Interagency Report NISTIR 6376, National Institute of Standards and Technology, Gaithersburg, MD, 1999.

${ }^{9}$ A. Aharoni, Phys. Rev. 119, 127 (1960).

${ }^{10}$ C. Abraham and A. Aharoni, Phys. Rev. 119, 127 (1960). 